\title{
GRAPPA Trainees Symposium 2016: A Report from the GRAPPA 2016 Annual Meeting
}

\author{
Ari Polachek, Carmel B. Stober, Bjorn Gudbjornsson, and Christopher T. Ritchlin \\ ABSTRACT. At the 2016 annual meeting of the Group for Research and Assessment of Psoriasis and Psoriatic \\ Arthritis (GRAPPA) in Miami, Florida, USA, a trainees symposium was held. Rheumatology and \\ dermatology trainees, engaged in psoriasis or psoriatic arthritis research, presented their work. This \\ report briefly reviews 6 oral presentations and 21 posters presented at that meeting. (J Rheumatol \\ 2017;44:661-7; doi:10.3899/jrheum.170140)
}

Key Indexing Terms: PSORIATIC ARTHRITIS PSORIASIS RHEUMATOLOGIST DERMATOLOGIST TRAINEE GRAPPA

The Group for Research and Assessment of Psoriasis and Psoriatic Arthritis (GRAPPA) held this year's trainees symposium at its 2016 annual meeting in Miami, Florida, USA. As in past years, rheumatology or dermatology trainees from North America, South America, and Europe who are current members of GRAPPA or who were nominated by GRAPPA members were invited to submit abstracts based on recent research in psoriatic arthritis (PsA) or psoriasis $1,2,3,4,5,6,7$. A total of 27 abstracts were submitted and ranked by a committee of reviewers. Six trainees with the highest-scored abstracts were invited to deliver oral presentations; all presented posters that outlined key aspects of their research. Dr. Christopher T. Ritchlin (Rochester, New York, USA) chaired the symposium in which GRAPPA members discussed the findings presented by trainees and suggested how they might further their current research projects.

\section{Oral Presentations}

Endocrine comorbidities in patients with PsA: A population-based case-controlled study (Amir Haddad, Haifa, Israel). PsA is associated with cardiovascular, ophthalmic, and gastrointestinal (GI) comorbidities. This retrospective,

From the University of Toronto, Toronto, Ontario, Canada; University of Cambridge, Cambridge, UK; Division of Allergy, Immunology, and Rheumatology, University of Rochester Medical Center, Rochester, New York, USA; Centre for Rheumatology Research, National University Hospital of Iceland, Reykjavik, Iceland.

As part of the supplement series GRAPPA 2016, this report was reviewed internally and approved by the Guest Editors for integrity, accuracy, and consistency with scientific and ethical standards.

A. Polachek, MD, Clinical and Research Fellow, University of Toronto; C.B. Stober, MBChB, PhD, University of Cambridge; B. Gudbjornsson, $M D$, PhD, Professor, Centre for Rheumatology Research, National University Hospital of Iceland; C.T. Ritchlin, MD, MPH, Professor of Medicine, Division of Allergy, Immunology, and Rheumatology, University of Rochester Medical Center.

Dr. Polachek and Dr. Stober are joint first authors.

Address correspondence to Dr. C.B. Stober, NIHR Academic Clinical Lecturer, University of Cambridge, Department of Medicine,

Box 157, Addenbrooke's Hospital, Cambridge CB2 OQQ, UK.

E-mail:cbs24@medschl.cam.ac.uk cross-sectional study was performed using the database of the largest healthcare provider in Israel, evaluating data collected between 2000 and 2013, with a goal of finding the prevalence and association of endocrine comorbidities in patients with PsA.

The study included 3161 patients with PsA, with a mean age of $58.36 \pm 15.42$ years, 1687 women $(53.4 \%)$ and 31,610 controls (matched for age and sex). The patients with PsA had a significantly higher prevalence of hypothyroidism ( $12.7 \%$ vs $8.6 \%, \mathrm{p}<0.0001)$, Cushing disease $(0.3 \%$ vs $0.1 \%, \mathrm{p}<0.0001)$, osteoporosis $(13.2 \%$ vs $9.1 \%, \mathrm{p}<0.001)$, and diabetes mellitus (DM; $27.9 \%$ vs $20.7 \%$, p < 0.0001 ) compared with the control group. Multivariate regression analysis models, adjusted for relevant confounding factors, showed higher OR in patients with PsA for the presence of hypothyroidism (OR 1.61, 95\% CI 1.43-1.80), DM (OR $1.30,95 \%$ CI 1.18-1.42), Cushing disease (OR 3.79, 95\% CI 1.64-8.77), and osteoporosis (OR 1.50, 95\% CI 1.33-1.70). Dr. Haddad recommended that awareness of these comorbid disease associations by physicians would facilitate delivery of more comprehensive medical care to patients with PsA.

The association between sonographic enthesitis and radiographic damage in PsA (Ari Polachek, Toronto, Ontario, Canada). Enthesitis is a common clinical finding and a key pathogenic feature in PsA. Ultrasound is a preferred method to assess enthesitis. In this cross-sectional analysis of 193 patients, the association between sonographic enthesitis and the severity of radiographic features of joint damage was examined. The MAdrid Sonography Enthesitis Index (MASEI) scoring system was used to quantify the extent of sonographic entheseal abnormalities. Radiographic joint damage in the peripheral joints and spine was assessed using the modified Steinbrocker score and features of joint ankylosis and arthritis mutilans. Radiographic damage in the axial skeleton was assessed using the modified New York criteria for sacroiliitis and the modified Stoke Ankylosing Spondylitis Spine Score (mSASSS).

Multivariate regression analyses found an association

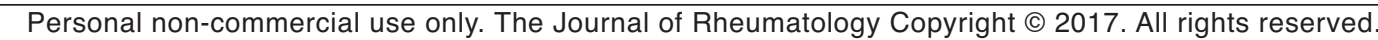


between higher MASEI scores and peripheral joint damage: modified Steinbrocker score $(\beta 0.87, \mathrm{p}<0.0001)$, periostitis $(\mathrm{OR} 1.05, \mathrm{p}=0.0006)$, joint ankylosis (OR 1.07, $\mathrm{p}=0.0007)$, and arthritis mutilans (OR 1.06, $\mathrm{p}=0.007)$. Similarly, multivariate analyses revealed an association between higher MASEI scores and axial damage as measured by the mSASSS $(\beta 0.14, p=0.001)$. The authors concluded that the severity of sonographic enthesitis is a marker of radiographic peripheral and axial joint damage in PsA, suggesting a potential involvement of enthesitis in the pathogenesis of articular damage in PsA.

Higher frequency and severity of coronary plaques on coronary computerized tomography (CT) angiography in patients with PsA without symptoms of coronary artery disease $(C A D)$ compared with controls (Agnes Szentpetery, Dublin, Ireland). Dr. Szentpetery evaluated the presence, extent, and composition of coronary plaques as measured by coronary CT angiography (CCTA) in PsA without symptoms of CAD in 50 patients with PsA [ 25 with metabolic syndrome (Metsy) and 25 Non Metsy (NMetsy)] and 25 controls without CAD (age- and sex-matched) who underwent 64-slice CCTA. Several plaque characteristics were assessed: plaque localization, plaque type [calcified, mixed (MP) and non-calcified plaque], plaque volume (PV), the number of segments with plaque [segment involvement score (SIS)], and the segment stenosis score (SSS).

They found that $78 \%$ of PsA versus $44 \%$ of controls had plaques $(p=0.007)$, and the number of affected coronary vessels were higher in PsA ( $p=0.015)$. The MP and MP volume was higher in patients with PsA than controls $(\mathrm{p}=$ 0.006 ). Mean PV, SIS, and SSS were higher in patients with PsA ( $p=0.002, p=0.032, p=0.004$, respectively). Skin- and joint-related variables and CCTA measurements were similar in Metsy versus NMetsy. Maximum C-reactive protein (CRP) correlated significantly with PV, SIS, and SSS in PsA. Disease duration, maximum tender joint count (TJC), and maximum CRP correlated significantly with the number of affected coronary vessels $(\mathrm{p}=0.034, \mathrm{p}=0.049, \mathrm{p}=0.022$, respectively). These findings suggest that PsA may result in accelerated coronary plaque formation, independent of metabolic disease.

Cutaneous microbiota in psoriasis and PsA (Julia Manasson, New York, USA). The host microbiota is considered a potential trigger of host autoimmunity, especially regarding the spondyloarthritides, including PsA. Dr. Manasson compared the microbiota identified from skin swabs taken from psoriatic plaques or unaffected skin of either patients with psoriasis only $(n=29)$ or PsA $(n=62)$. Using the Quantitative Insights into Microbial Ecology pipeline, 16s rRNA sequences were analyzed; alpha diversity plots were generated and Unifrac analysis was used to measure beta diversity and perform principal component analysis of cutaneous bacterial species.

Skin samples obtained from individuals with psoriasis had a lower number of operational taxonomic unit reads compared with samples from patients with PsA. Second, there was a difference in the clustering of microbial communities between individuals with psoriasis and PsA, driven by psoriatic lesions rather than unaffected skin. In particular, increases in specific genera were detected in psoriasis lesional skin including Bradyrhizobiaceae, Rahnella, Parvibaculum, and unclassified Prevotellaceae, while Lactobacillus was overrepresented in PsA lesional skin. The group had previously demonstrated reduced bacterial diversity in the guts of patients with psoriasis and PsA, with PsA microbiota being less diverse. Further in-depth analysis may facilitate better understanding of these differentiating taxonomic features and their involvement in the pathogenesis of the psoriatic spectrum of disease.

Janus Kinase and Signal Transducer and Activator of Transcription (JAK-STAT) signaling system regulates the Th17 cells in PsA (Kanika Sood and Suravi Raychaudhuri, Mather, California, USA). Genome-wide association studies have demonstrated the importance of the interleukin (IL)-23/Th17 axis in the pathogenesis of psoriasis and PsA. Both TYK2 and JAK2 are recruited to the IL-23 receptor, promoting signaling through STAT3 and STAT4. This study analyzed the involvement of JAK-STAT signaling in Th17 cells in PsA, and whether tofacitinib, an inhibitor of JAK-1, -2 , and -3 , inhibited IL-23 receptor-mediated JAK-STAT signaling.

Peripheral blood mononuclear cells (PBMC) and synovial fluid MC (SFMC) were obtained from 15 patients with active PsA who were naive to disease-modifying antirheumatic drugs (DMARD), and 15 healthy controls. The authors used Western blotting to demonstrate IL-23-mediated JAK2, TYK2, and STAT3 phosphorylation in CD3+ T cells, with JAK2 and STAT3 phosphorylation being inhibited by tofacitinib. Coincubation of CD4+CD11a+CD45RO+ memory T cells with IL-23 increased the expression of IL-17+ cells to $30 \pm 4.5 \%$ in SFMC and $18 \pm 3.8 \%$ in PBMC from patients with PsA compared to just $5 \pm 0.7 \%$ in healthy donor PBMC. Tofacitinib reduced the proportion of CD4+CD11a+CD45RO+IL-17+ cells (from $18 \pm 3.8 \%$ to $5 \pm 2.0 \%$ ), and also inhibited proliferation, as assessed by carboxyfluorescein succinimidyl ester assay. Tofacitinib has shown efficacy in a phase III study in PsA, which may provide an alternative therapeutic option for both PsA and psoriasis in the future by targeting IL-23mediated signaling.

Is GM-CSF release by Th17 cells important in the pathogenesis of PsA (Carmel Stober, Cambridge, UK)? Studies analyzing Th17 pathogenicity in mouse models of inflammatory disease such as experimental autoimmune encephalomyelitis have identified at least 2 Th17 subsets: nonpathogenic T cells that coproduce IL-17A and IL-10 and express the transcription factor ROR- $\gamma \mathrm{t}$, or cells that make IL-17A, interferon (IFN)- $\gamma$, and granulocyte-macrophage colony-stimulating factor (GM-CSF), express T-bet

Personal non-commercial use only. The Journal of Rheumatology Copyright @ 2017 . All rights reserved. 
and ROR- $\gamma \mathrm{t}$, and are augmented by IL-23. Dr. Stober sought to identify whether GM-CSF production by pathogenic $\mathrm{T}$ cells was also a feature observed in human subjects with PsA.

Results revealed that GM-CSF levels were higher and IFN- $\gamma$ levels lower in subjects with PsA relative to healthy donors ( $<<0.01)$, and there was enrichment of GM-CSFproducing cells in SFMC. Both naive and memory CD4+ $T$ cells increased IL-17A release whereas GM-CSF release was reduced by IL-23, which differed from previous murine studies. Further, there were more peripheral CD4+GM-CSF+ and fewer CD4+GM-CSF+IFN- $\gamma+$ cells in patients with PsA. About 50\% of GM-CSF+ T cells expressed markers of Th17 (CCR6+CD161+IL-23R+); therefore, the authors hypothesized that enrichment of CD4+GM-CSF+IFN- $\gamma$ - cells in PsA may reflect this pathogenic subset observed in mice.

\section{Poster Presentations}

M.L. Acosta Felquer (Buenos Aires, Argentina and Dublin, Ireland) defined remission (REM) and different disease activity states [including low (LDA), moderate (MoDA), or high (HDA) disease activity] in PsA based on the Composite Psoriatic Disease Activity Index (CPDAI). Data from 87 patients with PsA in a daily clinical practice database were assigned to these disease activity states according to the clinical Disease Activity Index for PsA (cDAPSA). CPDAI differed significantly between patients classified as REM, LDA, MoDA, or HDA $(p<0.001)$ cDAPSA. Based on the distributions of CPDAI in these groups, the cutoff values were $<2$ for REM, $\geq 2$ and $<4$ for LDA, $\geq 4$ and $<7$ for MoDA, and $\geq 7$ for HDA. With these cutoff values, $22 \%$, $44 \%, 14 \%$, and $19 \%$ of the patients were in REM, LDA, MoDA, and HDA, respectively. None of the patients in remission by CPDAI changed DMARD at the index visit, while $33 \%, 42 \%$, and $53 \%$ of patients in LDA, MoDA, and HAD, respectively, did undergo a treatment change. The author concluded that CPDAI constitutes a disease-specific, validated, and feasible tool for PsA assessment.

Thales Pereira de Azevedo (Rio de Janeiro, Brazil) demonstrated that the histological pattern and immunoreactivity to anti-IL-6, IL-17, and IL-23 in the inflammatory response of geographic tongue (GT) is similar to psoriasis. Tissue samples from GT and skin lesions of 35 subjects (20 with GT and 15 with psoriasis and GT) were taken. The histopathological results showed predominance of low intensity superficial and papillary mononuclear inflammatory infiltrate in both peripheral and central areas of both groups. The immunohistochemical study with anti-IL-6, anti-IL-17, and anti-IL 23 showed similar cytoplasmic immunoreactivity in psoriasis and GT, both in the epithelium and in the inflammatory infiltrate. IL-6 immunoreactivity was noted on keratinocytes at the epidermis lower third, which is proportional to the basal layer hyperplasia. IL-17 immunoreactivity was identified in the lower epidermis and IL-23 showed basal and suprabasal immunoreactivity, extending over superficial portions of the epithelium. Thus, the author concluded that the histopathologic and immunohistochemical features of GT are similar to those of psoriasis, reinforcing the possibility that GT represents an oral manifestation of psoriasis.

Hatice Sule Baklacioglu (Istanbul, Turkey) assessed the static and dynamic balances of the feet in patients with PsA and correlated the findings with clinical, functional, and radiological findings. State of balance was evaluated using the Berg Balance Scale, the Modified Clinical Test of Sensory Interaction and Balance, and the Unilateral Stance for static balance assessment, as well as the Step Up/Over and Tandem Walk tests for dynamic balance assessment (through the Neurocom Balance Master). Of the 100 subjects in the study, 50 patients with PsA who fulfilled the Classification Criteria for PsA (CASPAR) and 50 healthy controls, the PsA group had a significantly higher body mass index (BMI; $\mathrm{p}<0.05)$. The mean 28-joint Disease Activity Scores (DAS28) and Psoriasis Area Severity Index (PASI) scores of the patients with PsA were 3.45 and 7.80, respectively. According to specific surveys, $41.7 \%$ of patients with PsA had depression, $76 \%$ sleep disorders, and $88 \%$ fatigue, though there were no correlations between these variables and balance test results. The fear of falling and history of falling were not found to be associated with the balance variables. Although $90 \%$ of patients with PsA were diagnosed with foot deformities (based on radiographic results), these deformities did not correlate with foot function assessment and balance test results. Significant differences were found between patients with PsA and controls $(\mathrm{p}<0.05)$ for the "velocity tests, tandem walk test, and movement time of step up over test". The author concluded that balance disorders in patients with PsA may be independent from other findings and symptoms that arise during the course of the disease.

Mark Berman (Tel Aviv, Israel) evaluated the effect of pregnancy on disease activity in a retrospective study of 13 women with PsA who had 20 pregnancies, 19 of which resulted in live healthy babies. PsA activity was defined as no disease activity (no active synovitis), mild disease (up to 1 joint involved), or moderate to severe disease (more than 2 joints involved). No significant change in disease activity was noticed throughout pregnancy while a significant proportion of patients flared postpartum. In 10 pregnancies, biologic treatment [tumor necrosis factor- $\alpha$ (TNF- $\alpha)$ ] was discontinued close to pregnancy or during the first trimester. In this group, 4 patients (40\%) were classified as having mild to severe activity prior to pregnancy, and disease activity increased in $6(60 \%), 7(70 \%)$, and $10(100 \%)$ of pregnancies during the first and second trimesters and postpartum period, respectively. In 2 pregnancies where biologics were not stopped, no change in degree of disease activity was noticed. Interestingly, in the group of patients not receiving TNF- $\alpha$ (10 pregnancies), an improvement in disease activity was observed; the proportion of patients with mild to severe

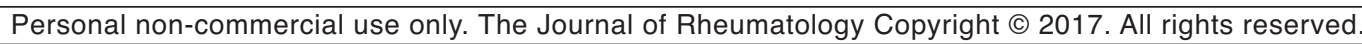


disease activity decreased from $100 \%$ close to pregnancy to $71 \%$ in the first and second trimesters and $43 \%$ in the third, while an increase to $86 \%$ was observed after pregnancy. Based on this small study, the author recommends continuing treatment with TNF- $\alpha$ blockers throughout pregnancy.

Musaab Elmamoun (Dublin, Ireland) correlated skin-related patient self-assessment questionnaires and skin scores assessed by physicians using a Web-based tool called the Measuring Outcome in PsA in 189 patients with PsA and 52 patients with psoriasis who had skin scores. The mean \pm SD Dermatology Quality of Life Index (DLQI) was 5.57 \pm 6.93 , Psoriasis Symptom Inventory (PSI) $7.36 \pm 8.68$, body surface area (BSA) $7.27 \pm 12.17$, and PASI $4.57 \pm 5.56$. There was strong correlation between the PASI and BSA ( $\mathrm{r}=$ $0.8502, \mathrm{p}<0.0001)$ and between the PASI and DLQI $(\mathrm{r}=$ $0.7256, \mathrm{p}<0.0001$ ), but only moderate correlation between the PASI and PSI $(r=0.5812, p<0.0001)$. There was strong correlation between the BSA and DLQI $(r=0.7048$, $p<$ $0.0001)$, but only moderate correlation between the BSA and PSI $(r=0.6246, p<0.0001)$. Finally, there was strong correlation between the DLQI and PSI $(r=0.7873, p<0.0001)$. These preliminary results suggest that the combination of BSA and DLQI might work just as well as the PASI and DLQI to accurately assess skin involvement in rheumatology clinics.

Victoria Furer (Tel Aviv, Israel) examined the prevalence of nonradiographic axial spondyloarthropathy (nr-axSpA) in a consecutively recruited observational cohort of PsA. All patients underwent clinical and laboratory evaluation, plain radiograph of the sacroiliac joints (SIJ), and magnetic resonance imaging (MRI) of the entire spine and SIJ. Radiographs were independently read by 2 musculoskeletal radiologists (New York criteria). MRI examinations were interpreted by a third musculoskeletal radiologist using the Association of SpondyloArthritis International Society (ASAS) criteria and global SIJ scoring. Full imaging sets were available for 41 patients (of 61 who were recruited) at the time of the interim analysis. All patients were HLA-B27negative. Interreader agreement between the 2 radiographic readers was good $(x=0.73)$. In $17 \%$ of the cohort, nr-axSpA was detected, while truly radiographic axSpA was detected in $35 \%$. Twelve percent of the patients demonstrated false-positive sacroiliitis by radiograph, not confirmed by MRI; these patients were older compared with others ( $\mathrm{p}=$ 0.078). MRI-positive sacroiliitis (ASAS/global) was detected in $31 \% / 44 \%$ patients, respectively. Extensive psoriasis, assessed by the PASI, was associated with global MRI/entire spine sacroiliitis presence $(\mathrm{p}=-0.0017$ and 0.044 , respectively). Clinical indices of peripheral and spinal disease activity did not correlate with MRI sacroiliitis. Biologic-naive patients demonstrated similar rates of sacroiliitis to those of patients exposed to biologic therapies.

Kardelen Gencer (Istanbul, Turkey) assessed the validity and reliability of the Jenkins Sleep Scale (JSS) in a Turkish population of 56 patients with PsA with a mean age of 42.7 (SD 11.8) years. The mean duration of disease and joint symptoms was 38.8 months (SD 42.7) and 61.4 months (SD 58), respectively. The Cronbach's alpha coefficient (assessing internal consistency) of the JSS was 0.832. All questions and answer choices of the JSS were well understood by patients, showing good face validity. The JSS had significant correlation with the Pittsburgh Sleep Quality Index (PSQI; $r=$ $0.510, p<0.0001)$, the Multidimensional Assessment of Fatigue $(\mathrm{r}=0.405, \mathrm{p}=0.002)$, and the Psoriatic Arthritis Quality of Life scales $(r=0.421, p=0.001$; convergent validity). The JSS had good correlation with 5 subgroups of the PSQI, but "sleep latency" and "need medication to sleep" subgroup correlations were not significant. The JSS correlated with age and disease duration, but correlation with duration of joint symptoms was not significant (divergent validity). The author concluded that the JSS is a valid and reliable instrument in patients with PsA in the Turkish population.

Charis Gn (Providence, Rhode Island, USA) presented the characteristics and outcome of patients who were followed in a rheumatology-dermatology clinic of a tertiary care medical center. In a retrospective chart review of 324 patient visits, a total of 167 new patients were identified. The majority $(68 \%)$ of patients were referred by either dermatology or rheumatology for further evaluation and management; diagnosis included psoriasis, PsA, systemic lupus erythematosus, cutaneous lupus, dermatomyositis, scleroderma, vasculitis, pyoderma gangrenosum, hidradenitis suppurativa, and mixed connective tissue disease. Forty-four patients $(26 \%)$ reported a history of a comorbid autoimmune condition such as inflammatory bowel disease, thyroid disease, or pernicious anemia. Because of their consultation in the combined dermatology-rheumatology clinic, 55 patients $(33 \%)$ received a change in diagnosis, and treatment was changed in 94 patients (56\%). Sixty-eight patients (41\%) had escalation of treatment, while $12(7 \%)$ had treatment reduction. The author concluded that patients with complex autoimmune diseases, including those with psoriasis and PsA, experienced benefits from co-management of their condition.

Jacqueline Greb (Boston, Massachusetts, USA) evaluated disease characteristics and treatment response variation in 191 patients with moderate to severe psoriasis, 58 with and 133 without rheumatologist-diagnosed PsA, in a retrospective analysis. The improvement in the Simple-Measure for Assessing Psoriasis Activity, which is the product of the mean physician's global assessment (PGA) $\times$ the BSA, was similar in both groups (with PsA and without PsA) with biologic monotherapy (46.2 vs $44.1, \mathrm{p}=0.74)$, traditional systemic monotherapy (62.29 vs $38.12, \mathrm{p}=0.22)$, and combination treatments $(64.62$ vs $52.71, \mathrm{p}=0.40)$ after 12 weeks. Patients with PsA receiving biologic monotherapy experienced a higher infection rate than patients without PsA $(0.57 \%$ vs

Personal non-commercial use only. The Journal of Rheumatology Copyright $\odot$ 2017. All rights reserved. 
$0.19 \%, \mathrm{p}=0.01)$. Patients with PsA experienced more adverse events (AE) associated with traditional systemic monotherapy than biologic monotherapy (3.25 vs 1.04 , p $=$ $0.001)$. The primary limitation was the relatively small PsA cohort. The author concluded that patients with moderate to severe psoriasis responded similarly to all treatments independent of PsA history. In addition, patients with PsA received more treatments including more biologic monotherapy prescriptions.

Tania Gudu (Paris, France) assessed the effect of PsA from the patient's perspective mapped to the International Classification of Functioning, Disability and Health (ICF). Publications with dimensions of health importance for patients with PsA were identified through a systematic literature review. Only studies related to effect from the patient's perspective were included. Eleven studies (13 articles) were included in the analysis, corresponding to 2893 patients. Twenty-five domains of health were cited, of which the ability to work/volunteer and social participation were the most cited. In total, 258 concepts were identified, of which $217(84.1 \%)$ could be linked to 136 different ICF categories; 41 concepts $(15.9 \%)$, mostly personal factors, primarily coping with the disease (21 concepts; $51.21 \%$ ), could not be linked. The most represented ICF component was activities and participation (42.6\%) rather than body functions (29.4\%) or body structures $(10.3 \%)$. Ten studies $(90.9 \%)$ reported impairments in ability to work/volunteer and social participation; 7 (63.6\%) reported problems with leisure activities, family and intimacy, pain, skin, and body image. The author concluded that PsA widely affects all aspects of patients' lives, particularly regarding activities and participation, reflecting the functional limitations and the psychosocial and societal burden of PsA.

Ariel Zohar (Haifa, Israel) evaluated the association between PsA and common GI diseases in 3161 patients with PsA identified in the database of Israel's largest healthcare provider between 2000 and 2013. For each patient with PsA, 10 patients of the same age and sex were randomly selected from the database to serve as the control group. The results showed that PsA was associated with Crohn disease (OR 2.4, $\mathrm{p}<0.0001$ ), ulcerative colitis (OR 2.1, $\mathrm{p}=0.001$ ), reflux esophagitis (OR 1.6, $\mathrm{p}<0.0001$ ), peptic ulcer disease (PUD; OR 1.5, p < 0.0001), and irritable bowel syndrome (IBS; OR $1.4, p=0.045)$. After controlling for known risk factors, the association remained significant between PsA and Crohn disease (OR 2.2, $\mathrm{p}<0.0001)$, ulcerative colitis (OR 1.9, $\mathrm{p}=$ 0.005 ), reflux esophagitis (OR 1.5, p < 0.0001), PUD (OR $1.3, \mathrm{p}<0.0001)$, and IBS. No significant association was found between PsA and celiac disease $(p=0.216)$. There were no differences in GI morbidities between patients with PsA who were never treated with biological and nonbiological DMARD and patients treated with DMARD. The author suggested that physicians treating patients with PsA should be aware of these associations.
Richard Holland (Bath, UK) performed a prospective, single-center cohort study of patients commencing TNF inhibitor (TNFi) therapy for PsA (July 2003-July 2015; n = 207, 104 men), in which TNFi survival and factors that may predict it were assessed. Sixty-three percent of patients received concomitant DMARD and 37\% monotherapy. Survival on the first agent decreased from $90.3 \%$ at 3 months to $74 \%$ at 12 months, and $64.8 \%$ at 24 months (median survival $59 \mathrm{mos})$. Reasons for discontinuation included secondary failure (27\%), side effects (16\%), infection (13\%), and primary failure $(13 \%)$. In multivariate analysis, male sex, concomitant methotrexate (MTX), and lower baseline TJC remained significant predictors of longer drug survival. Eighty patients received a second TNFi (26 secondary failure, 13 primary failure, $41 \mathrm{AE})$ and 30 patients a third TNFi (8 secondary failure, 7 primary failure, $15 \mathrm{AE}$ ). Median survival was 34 months and 18 months for a second and third TNFi, respectively. Persistence was $77 \%$ and $75 \%$ at 6 months, $65 \%$ and $56 \%$ at 12 months, and $52 \%$ and $46 \%$ at 24 months for the second and third TNFi, respectively, thus providing some validation for switching to another TNFi after failing the first.

Deep Joshipura (Boston, Massachusetts, USA) used data from the Consortium of Rheumatology Researchers of North America registry to compare findings in PsA and rheumatoid arthritis (RA). Studies of bone loss in PsA found that patients with erosions had significantly lower lumbar spine bone mineral density than those without erosions. Patients with PsA demonstrated higher rates of DM, elevated triglycerides, higher BMI, and more metabolic syndrome relative to RA. There were no differences in the rates of malignancy or cancer subtypes between PsA and RA cohorts. Biologics were used more frequently in PsA, while DMARD and prednisone were used more commonly in patients with RA. Abnormal liver enzymes were observed in $14 \%-35 \%$ of patients with RA or PsA receiving DMARD therapy, with a greater risk in those receiving simultaneous leflunomide and MTX ( $\geq 10 \mathrm{mg} /$ day); patients with PsA demonstrated a 3-fold increased risk of transaminase elevations than patients with RA, emphasizing the importance of monitoring for potential hepatotoxicity. Surprisingly, similar levels of disease severity and radiographic progression were observed in PsA versus RA, thus underlining the importance of early and aggressive treatment in PsA.

Sibel Ureyen (Sakarya, Turkey) assessed the performance of 3 different classification systems for inflammatory back pain (IBP; Calin, Berlin, and ASAS) in a large multicenter PsA cohort using data from the Psoriatic Arthritis Registry of Turkey (PsART), a Web-based prospective registry. Of the 267 patients (154 women, 26.6\%) with lower back pain, the fulfillment of criteria for IBP was comparable for Berlin $(\mathrm{n}=197,73.8 \%)$ and Calin $(\mathrm{n}=206,77.2 \%)$, but lower for ASAS ( $\mathrm{n}=156,58.4 \%)$; there was moderate agreement between criteria $(\kappa=0.536-0.626)$. More than $80 \%$ of patients developed back pain prior to age 40 , had back pain

Personal non-commercial use only. The Journal of Rheumatology Copyright (C) 2017. All rights reserved. 
for $>3$ months, and morning stiffness for $>30 \mathrm{~min}$, whereas $<50 \%$ reported nocturnal awakening. On evaluating clinical variables (Bath Ankylosing Spondylitis Disease Activity Index, Bath Ankylosing Spondylitis Functional Index, patient's global assessment, PGA, pain, fatigue, CRP, erythrocyte sedimentation rate) in those fulfilling criteria or not, CRP was significantly higher in patients fulfilling the Calin criteria, and fatigue was significantly worse in patients meeting the ASAS or Berlin criteria. Finally, $42 \%$ of patients with IBP had plain radiographs or MRI of sacroiliac joints $(n=113 / 267)$, and $78 \%$ of these fulfilled axSpA criteria according to the ASAS imaging arm. There was $72 \%$ agreement with the Calin IBP criteria and imaging, $71 \%$ for Berlin IBP, and $66 \%$ for ASAS IBP criteria and imaging. The authors concluded that the Calin IBP performed better than the ASAS for identifying patients with a diagnosis of axial PsA, and this was particularly true for women.

Levent Kilıç (Ankara, Turkey) presented data from the PsART Study Group to understand how disease duration affects the type of joint manifestations in PsA. They analyzed 799 patients (67\% women) from 32 centers with mean (SD) psoriasis duration of 15.1 years (11.4) and mean (SD) PsA duration 5.5 years (6.6). PsA subtypes were identified as polyarticular $(n=317,39.6 \%)$, oligoarticular $(286,35.7 \%)$, monoarticular $(29,3.6 \%)$, axial $(102,12.8 \%)$, and distal interphalangeal (DIP; 63, 7.9\%). Disease duration was $\leq 1$ year in 212 patients $(26.6 \%), 1-5$ years in $290(36.3 \%)$, and $\geq 5$ years in $297(37.2 \%)$. Whereas the percentage of patients with polyarthritis increased with longer disease duration (21 up to $47 \%, \mathrm{p}<0.001$ ), DIP involvement remained stable, and axial, monoarticular, and oligoarticular disease reduced with time. The authors acknowledge that polyarticular subtypes were more likely to be prevalent in established disease and hypothesized that the decrease in axial disease could result from the cross-sectional design of the data, because patients with axial disease had less frequent followups.

Megan Noe (Pennsylvania, USA) used data from a primary care medical record database in the United Kingdom, The Health Improvement Network, to determine the prevalence of hepatitis $\mathrm{C}$ virus (HCV) infection and incidence of hepatic decompensation in patients with psoriasis compared with matched controls. A subanalysis was performed in a smaller cohort, the Incident Health Outcomes and Psoriasis Events (iHOPE) cohort, consisting of patients with psoriasis with data collected on BSA involvement. In 113,647 patients with psoriasis and 564,562 matched controls, the prevalence of $\mathrm{HCV}$ was $0.14 \%$ and $0.11 \%$, respectively. There was no difference in the OR of HCV infection or hepatic decompensation in patients with psoriasis compared with healthy controls. In the iHOPE cohort, the highest prevalence of $\mathrm{HCV}$ infection was seen in patients with mild disease $(<3 \%$ BSA), with a trend toward decreased prevalence of $\mathrm{HCV}$ infection in patients with moderate (3\%-10\% BSA) and severe (>10\% $\mathrm{BSA})$ disease. This trend toward decreased
$\mathrm{HCV}$ prevalence in patients with increased psoriasis BSA has not previously been identified and further research is necessary to determine whether the immunological and/or genetic changes present in patients with psoriasis provide a protective effect against $\mathrm{HCV}$ infection.

Elena Pezzolo (Verona, Italy) used a cross-sectional study of patients with moderate to severe chronic plaque psoriasis ( $n=281)$ and age- and sex-matched controls $(n=266)$ to estimate the prevalence and risk of chronic kidney disease (CKD) in patients with psoriasis. Glomerular filtration rate (GFR) was assessed by CKD Epidemiology Collaboration equation, the most accurate GFR-estimating equation to be evaluated in different large populations based on serum creatinine, age, sex, and race. The authors examined the modification of GFR in different treatment cohorts and the effect of comorbidities on the risk of CKD. Prevalence of CKD at baseline was $5.6 \%$ of patients with psoriasis and $0.3 \%$ of controls $(\mathrm{p}<0.01)$. After adjustment for CKD risk factors, moderate to severe psoriasis was associated with an increased risk of CKD (OR 1.04, 95\% CI 1.02-1.06). GFR did not change in patients receiving MTX or biologics, but cyclosporine (CSA) treatment resulted in a decrease in GFR from $94 \pm 17 \mathrm{ml} / \mathrm{min} / 1.73 \mathrm{~m}^{2}$ (baseline) to $78 \pm 22$ $\mathrm{ml} / \mathrm{min} / 1.73 \mathrm{~m}^{2}$ (after $6 \mathrm{mos} ; \mathrm{p}=0.01$ ). Twenty-six percent of patients receiving CSA $(3.5 \mathrm{mg} / \mathrm{kg} / \mathrm{day})$ developed estimated GFR $<60 \mathrm{ml} / \mathrm{min}$ after 6 months of continuous treatment, and serum creatinine increased from $0.8 \pm 0.1$ $\mathrm{mg} / \mathrm{dl}$ (baseline) to $1.1 \pm 0.3 \mathrm{mg} / \mathrm{dl}$ (Mo 6). The group proposed that moderate to severe psoriasis is associated with moderate to advanced CKD independent of traditional risk factors for renal dysfunction, and suggested screening and monitoring for CKD in this patient cohort, including testing for proteinuria and GFR.

Eugenia Saldarriaga (Bogotá, Colombia) evaluated a number of serum biomarkers as potential candidates for predicting disease activity or remission in 77 patients with PsA attending rheumatology or dermatology clinics (55\% men, mean age 54 yrs). Serum biomarkers included IL-17, IL-21, IL-23, matrix metalloprotease (MMP)-3, and CRP, and were compared using the DAPSA. MMP-3 was the biomarker most able to discriminate patients with active disease and those with remission, using either the likelihood ratio or the area under the receiver-operating characteristic curve. The group also examined a number of logistic predictive models for disease activity and remission in patients with PsA using DAPSA, MMP-3, and CRP. The group stated that a larger sample size would be required to validate their model.

Jordan M. Thompson (Providence, Rhode Island, USA) developed a revised and more comprehensive version of the original Psoriasis Screening Tool (PST), the PST-2, which included a psoriasis phenotype assessment, questions on treatment and perception of adequate treatment, and questions to assess the severity of a patient's disease at its worst, using BSA involvement as an indicator. Questions

Personal non-commercial use only. The Journal of Rheumatology Copyright @ 2017. All rights reserved. 
were included to assess the history of treatment, including detail of the type and duration of treatment. The PST-2 was piloted by the study group in dermatology clinics, and improvements on the PST include validating the date of diagnosis, measuring self-reported severity, identifying multiple psoriasis phenotypes, and identifying data on therapeutics and treatment history. When applied to larger cohorts, this should improve data collection, enable better characterization of risk factors and comorbidities, and serve as a holistic determination of a patient's severity of disease and previous treatments.

Desirée Valkinir (São Paulo, Brazil) analyzed whether the expression of Toll-like receptors (TLR)-2 and -4 were increased on monocytes in patients receiving anti-TNF therapy who developed new onset of psoriasis. Fifteen patients ( 10 women; mean age $45 \pm 14.03$ yrs, range $14-65$ yrs) and 15 age- and sex-matched healthy controls were included. Six received infliximab and 9 adalimumab, and the underlying conditions were Crohn disease $(n=6)$, ankylosing spondylitis $(n=6)$, RA $(n=2)$, and juvenile idiopathic arthritis $(n=1)$. Duration of anti-TNF therapy was $20 \pm 12.94$ months (range 0.5-84) prior to new-onset psoriasis. Patients had higher TLR- 2 expression on monocytes $(589 \pm 302 \mathrm{vs}$ $172 \pm 131, \mathrm{p}=0.023)$, but not on neutrophils $(7772 \pm 3271$ vs $5994 \pm 2920, p=0.33$ ), compared with controls, whereas there was no difference in the expression of TLR-4. The higher expression of TLR-2 on patient monocytes with anti-TNF-induced psoriasis may reinforce the idea that gram-positive bacteria induce higher inflammatory responses in this clinical manifestation, thereby triggering the innate immune system.

Leonieke van Mens (Amsterdam, the Netherlands) analyzed whether minimal disease activity (MDA) criteria were achieved in patients documented as having quiescent disease according to the treating rheumatologist. In a cross-sectional study of 250 patients fulfilling CASPAR with quiescent disease (i.e., the treating rheumatologist did not alter treatment), 88 (35\%) of the 250 patients with PsA did not fulfill MDA criteria (MDA-). There were no differences in treatment use between MDA- and MDA+. High TJC, visual analog score (VAS) pain, and VAS PGA most frequently contributed to failure to achieve MDA (not achieved in $83 \%, 82 \%$, and $80 \%$, respectively). Objective signs of disease activity were also more prevalent in the MDA- group: swollen joint count $>1$ in $35 \%$ versus $7 \%$ $(\mathrm{p}<0.001)$, enthesitis $>1$ in $14 \%$ versus $3 \%(\mathrm{p}=0.002)$, and PASI $>1$ in $43 \%$ versus $26 \%(\mathrm{p}=0.002)$. Patient-reported outcome (PRO) scores (Health Assessment Questionnaire, DLQI, and Work Productivity and Activity Impairment/Medical Outcomes Study Short Form-36 physical component score) were also significantly worse in the MDAversus MDA+ group. Because failure to achieve MDA was associated with worse PRO scores, the authors concluded that further research should evaluate whether treatment adjustments may be beneficial for this patient group with residual disease activity.

GRAPPA members were enthusiastic in their appreciation of the trainees' work and encouraged them to continue. The next GRAPPA Trainees Symposium will be held in July 2017 in Amsterdam, the Netherlands.

\section{REFERENCES}

1. Coates LC, Ritchlin CT. GRAPPA Trainees Symposium 2009: a report from the GRAPPA 2009 annual meeting. J Rheumatol 2011;38:526-9.

2. Ritchlin CT. GRAPPA Trainees Symposium 2010: a report from the GRAPPA 2010 annual meeting. J Rheumatol 2012;39:394-7.

3. Ash Z, Ritchlin CT. GRAPPA Trainees Symposium 2011: a report from the GRAPPA 2011 annual meeting. J Rheumatol 2012;39:2184-8.

4. Garg N, Touma Z, Ritchlin CT. GRAPPA Trainees Symposium 2012: a report from the GRAPPA 2012 annual meeting. J Rheumatol 2013;40:1413-8.

5. Szentpetery A, Johnson MA, Ritchlin CT. GRAPPA Trainees Symposium 2013: a report from the GRAPPA 2013 Annual Meeting. J Rheumatol 2014;41:1200-5.

6. Eder L, Tillett W, Ritchlin CT. GRAPPA Trainees Symposium 2014: a report from the GRAPPA 2014 Annual Meeting. J Rheumatol 2015;42:1016-20.

7. Milliken M, Generali E, Marin J, Ritchlin CT. GRAPPA Trainees Symposium 2015: a report from the GRAPPA 2015 Annual Meeting. J Rheumatol 2016;43:952-8. 